Open Access

\title{
Birth of a child with trisomy 9 mosaicism syndrome associated with paternal isodisomy 9: case of a positive noninvasive prenatal test result unconfirmed by invasive prenatal diagnosis
}

\author{
Jingmei Ma', David S. Cram², Jianguang Zhang ${ }^{2}$, Ling Shang ${ }^{2}$, Huixia Yang ${ }^{1 *}$ and Hong Pan ${ }^{1 *}$
}

\begin{abstract}
Background: Non-invasive prenatal testing (NIPT) is currently used as a frontline screening test to identify fetuses with common aneuploidies. Occasionally, incidental NIPT results are conveyed to the clinician suggestive of fetuses with rare chromosome disease syndromes. We describe a child with trisomy 9 (T9) mosaicism where the prenatal history reported a positive NIPT result for T9 that was unconfirmed by conventional prenatal diagnosis.

Methods: NIPT was performed by low coverage whole genome plasma DNA sequencing. Karyotyping and fluorescent in situ hybridization (FISH) analysis with chromosome $9 p$-ter and $9 q$-ter probes was used to determine the somatic cell level of T9 mosaicism in the fetus and child. Quantitative fluorescent PCR (Q-PCR) of highly polymorphic short tandem repeat (STR) chromosome 9 markers was also performed to investigate the nature of the T9 mosaicism and the parental origin.

Results: A 22 month old girl presented with severe developmental delay, congenital cerebral dysplasia and congenital heart disease consistent with phenotypes associated with T9 mosaicism syndrome. Review of the prenatal testing history revealed a positive NIPT result for chromosome T9. However, follow up confirmatory karyotyping and FISH analysis of fetal cells returned a normal karyotype. Post-natal studies of somatic cell T9 mosaicism by FISH detected levels of approximately $20 \%$ in blood and buccal cells. Q-PCR STR analysis of family DNA samples suggested that the T9 mosaicism originated by post-zygotic trisomic rescue of a paternal meiotic II chromosome 9 non-disjunction error resulting in the formation of two distinct somatic cell lines in the proband, one with paternal isodisomy 9 and one with T9.
\end{abstract}

Conclusion: This study shows that NIPT may also be a useful screening technology to increase prenatal detection rates of rare fetal chromosome disease syndromes.

Keywords: Noninvasive prenatal testing, Prenatal diagnosis, Karyotyping, Fluorescent in situ hybridization, Quantitative fluorescent PCR, Trisomy 9 mosaicism syndrome, Isodisomy 9, Uniparental disomy

\footnotetext{
* Correspondence: yanghuixia@bjmu.edu.cn; panmuren@263.net

${ }^{1}$ Department of Obstetrics, Peking University First Hospital, No.1 Xianmen

Street, Xicheng District, Beijing 100034, China

Full list of author information is available at the end of the article
} 


\section{Background}

Prenatal diagnosis by chorionic villous sampling or amniocentesis is the gold standard method for detection of fetal chromosomal abnormalities during pregnancy. With the advent of next generation sequencing technologies, it is now possible to detect fetal chromosomal abnormalities by massively parallel sequencing of the cell free DNA in the maternal circulation which contains on average $10-20 \%$ of fetal DNA during the second trimester [1]. Several large prospective trials $[2,3]$ have demonstrated that noninvasive prenatal testing (NIPT) is reliable and accurate with false positive and negative rates in the range of $0.1-0.2 \%$. Nonetheless, as the fetal DNA tested originates from the placental trophoblasts, it is strongly recommended that all positive NIPT results are confirmed by invasive prenatal testing [4]. While NIPT currently focuses on detection of common fetal autosomal aneuploidies such as T21, T18 and T13 as well as sex chromosomal aneuploidies, there has been research reports of positive results including T9 [5] and T2 [6], suggesting that NIPT may also have clinical utility for detection of rarer fetal trisomies. In this study, we present a confounding case of T9 mosaicism that was originally detected by NIPT, but was not confirmed by traditional invasive prenatal diagnosis methods.

\section{Case presentation}

A 22 month old girl presented at our genetic counselling clinic at the Peking University First Hospital with severe motor and intellectual disability, recurrent respiratory infection and failure to thrive. Physical examination revealed severe growth retardation with a weight of $7.5 \mathrm{~kg}$ ( $<3$ rd centile $10.9 \mathrm{~kg}$ ), height of $62 \mathrm{~cm} \mathrm{(<3rd} \mathrm{centile}$ $88.9 \mathrm{~cm})$ and head circumstance of $40 \mathrm{~cm}(<3 \mathrm{rd}$ centile $50 \mathrm{~cm}$ ). She displayed moderate to severe hypertonia. Dysmorphic features were visibly evident, including microcephaly, craniofacial asymmetry, long face, ocular hypertelorism, short and upward slanting palpebral fissures, short frenulum of the upper lip, high arched palate, low-set ears and abnormal nasal features including a prominent nasal bridge with a short root, a small fleshy tip and slit-like nostrils (Fig. 1). X-ray examination also revealed scoliosis. At 22 months of age, her developmental milestones were only equivalent to normal children of 7-9 months of age. She could not follow obvious movements with her eyes and struggled to hold her head up. The parents indicated that she had not developed any form of speech.

In review of the pregnancy history, maternal serum screening at 15 weeks gestation revealed a risk for the Down Syndrome of 1 in 200. Based on this risk, the couple elected to have confirmatory NIPT at 16 weeks. Chromosome (Chr) z-scores were 0.62 (Chr21),-0.27 (Chr18) and 0.016 (Chr13), all within the normal z-score range $(-3<\mathrm{z}<3)$. However, the $\mathrm{z}$-score for Chr9 (12.92) was outside the normal range, suggestive of T9. After calculation of copy number, the percentage of T9 was 30.1 \% (Fig. 2a), suggesting T9 mosaicism. The couple's clinician was informed about the possibility of a T9 mosaic fetus, prompting further prenatal investigation.

No structural anomalies in the second trimester were evident by ultrasound. However, at 30 gestational weeks, intrauterine growth restriction and lateral cerebral ventriculomegaly (right, $14 \mathrm{~mm}$ ) was observed. An echocardiography revealed no cardiac abnormalities. Further invasive prenatal diagnosis by percutaneous umbilical blood sampling was therefore performed at $34^{+2}$ gestational weeks in order to confirm the suspected T9 mosaicism or the presence of other possible chromosomal abnormalities. The fetal karyotype determined by analysis of 20 metaphase cells was 46, XX. Testing of both parents revealed normal peripheral blood karyotypes. No further molecular genetic testing was undertaken.
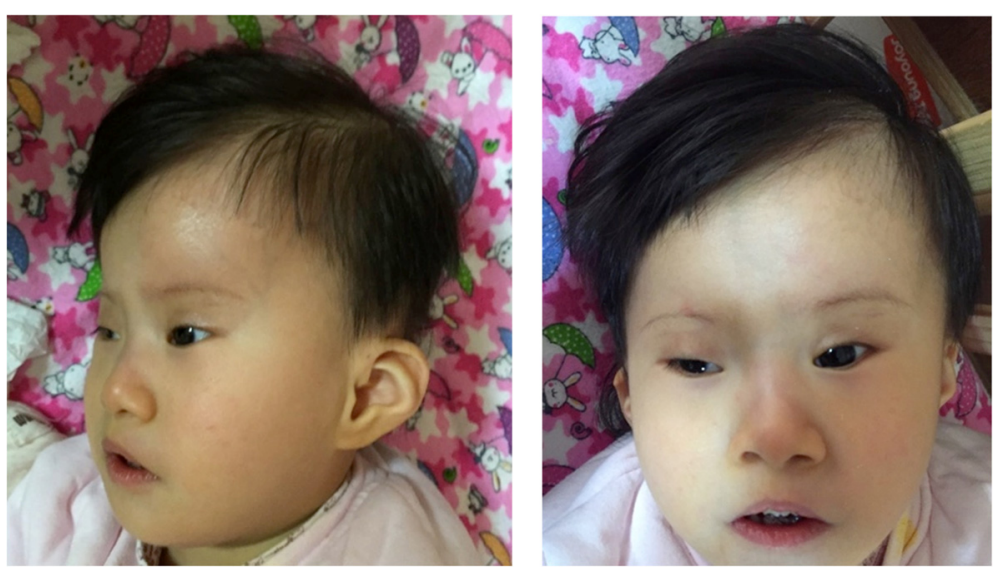

Fig. 1 Dysmorphic craniofacial features of the proband with trisomy 9 mosaicism syndrome 


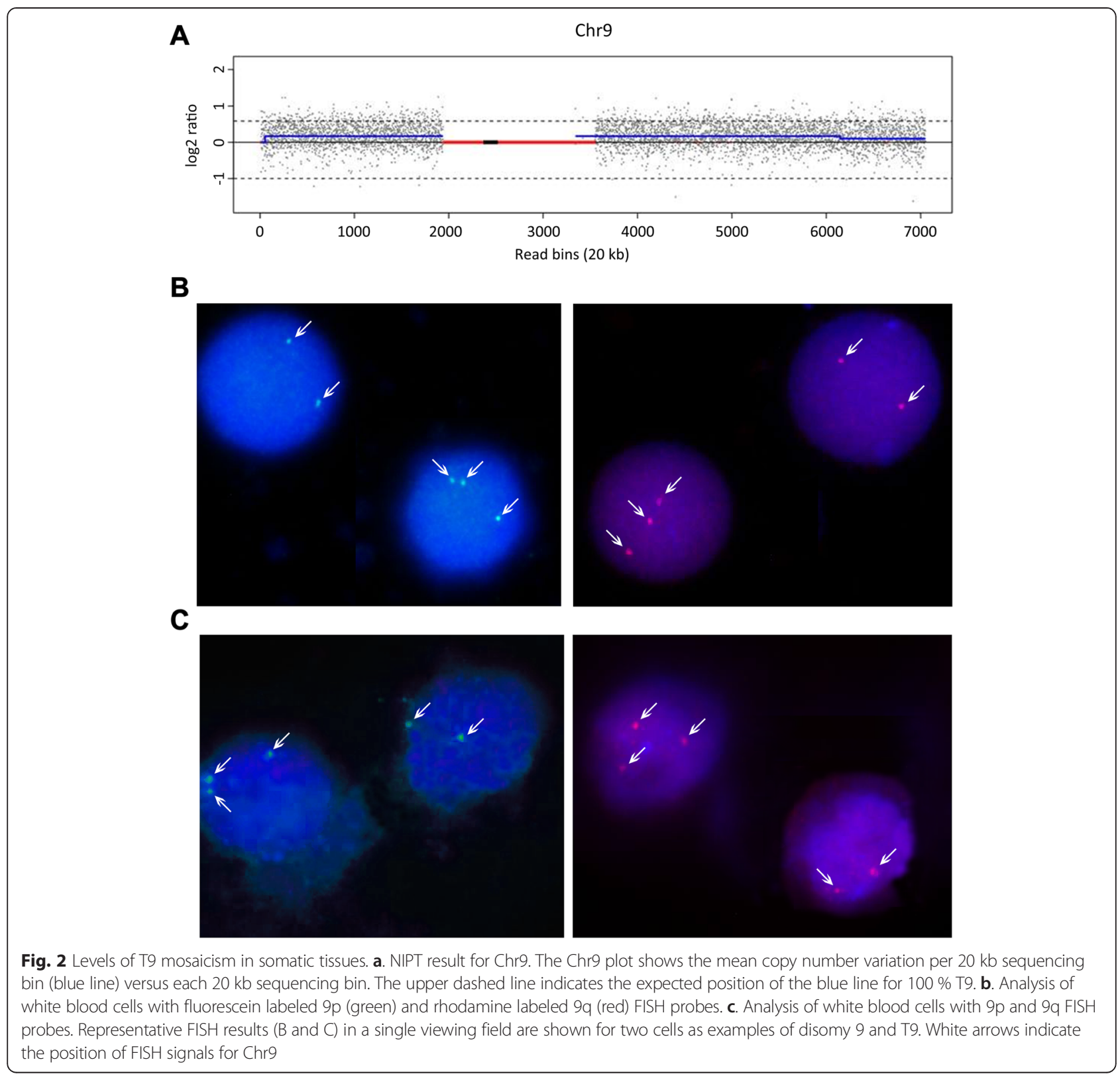

The girl was born by vaginal delivery at 41 weeks with a birth weight of $1960 \mathrm{~g}$, length of $45 \mathrm{~cm}$ and head circumstance of $30 \mathrm{~cm}(<5$ th centile $36 \mathrm{~cm})$. The newborn was referred to the Pediatrics department. Following ultrasound and MRI assessment, the final clinical diagnosis was congenital cerebral dysplasia, intracranial hemorrhage, thrombocytopenia and congenital heart disease (ventricular septal defect, patent ductus arteriosus). Further genetic testing was recommended.

The parental guardians of the child provided written consent to conduct post-natal genetic testing of the child at 22 months of age. Peripheral blood karyotyping of 200 metaphase cells identified 199 cells with a normal
46, XX karyotype and one cell with an abnormal 47, XX, +9 karyotype. Based on guidelines and recommendations of the International System for Human Cytogenetics Nomenclature [7], this karyotype should normally be reported as 46, XX. To investigate the presence of T9 mosaicism further, analysis of cultured blood cells with chromosome 9p-ter and 9q-ter FISH probes detected T9 in 1 of $100(1 \%)$ cells examined, consistent with karyotyping. However, interphase FISH analysis with the same probes applied to an uncultured peripheral blood smear revealed T9 in 19 of 100 (19\%) cells examined (Fig. 2b). To confirm T9 mosaicism in another tissue type, interphase FISH was also used directly to assess cheek 
buccal cells. T9 mosaicism was found in 17 of $100(17 \%)$ cells examined (Fig. 2c). On this basis, interphase FISH suggested that the somatic level of T9 mosaicism was approximately $20 \%$, differing slightly from the level of $30 \%$ in placental tissue detected by NIPT.

In order to investigate the nature of the $\mathrm{T} 9$ mosaicism and parental origin, we applied quantitative fluorescent PCR (QF-PCR) to analyse paternal, maternal and proband allelic patterns of short tandem repeat (STR) Chr9 markers. The alleles of STR markers D9S925, D9S1118 and D9S1121 were found to be informative for determining familial inheritance patterns (Fig. 3). All three Chr9 STR markers for the proband displayed a skewed bi-allelic pattern with allelic peak ratios of approximately 10:1 whereas the control D13S325 displayed a bi-allelic pattern with the expected peak ratio of approximately 1:1. Given that none of the Chr9 STR markers revealed a tri-allelic pattern typical of trisomy 9 , we deduced that the dominant Chr9 STR allelic peaks were consistent with inheritance of two identical paternal alleles (isodisomy) whilst the minor Chr9 STR allelic peaks were maternally inherited. Further, since the expected allelic peak ratio for a full T9 displaying a bi-allelic marker pattern is $2: 1$, then the observed peak ratios of approximately 10:1 suggest a level of around $20 \%$ T9 mosaicism, which was very similar to levels determined by FISH (Fig. 2b and 2c). Taken together, the proband inheritance pattern for Chr9 STR markers indicated the presence of two distinct cell lines in the child, one with paternal isodisomy 9 in approximately $80 \%$ of somatic cells and $\mathrm{T} 9$ in the remaining somatic cells.

\section{Discussion}

T9 syndrome and its related form T9 mosaicism syndrome are two extremely rare human chromosomal disorders. The first babies born with T9 [8] and T9 mosaicism [9] were reported in 1973. In the following four decades there have been sporadic reports in the medical literature of children with T9 [10] or T9 mosaicism associated with variable levels of somatic mosaicism ranging from $3 \%$ to $99 \%$ [11]. Children born with T9 usually die following birth or within one year of birth whereas those born with T9 mosaicism can survive into adulthood.

Based on data submitted to the tracking rare incidence syndromes (TRIS) project [11] and data published by the Rare Chromosomal Disorder Support Group (www.rarechromo.org), T9 mosaicism is generally associated with a low birth weight and a wide spectrum of developmental disabilities, abnormal physical features and organ pathologies, although the overall disease phenotype can vary widely between individuals. The main developmental disabilities reported are slow body growth, learning difficulties and delayed communication and speech. Characteristic physical features include microcephaly, loose joints, craniofacial abnormalities such as low set ears, cleft palate as well as skeletal abnormalities of the skull, hands and feet. Multiple tissue pathologies are common and can involve different organ systems such as the heart, kidneys, brain and CNS, the genital and urinary tract, muscles (hypertonia) and digestive tract. In the T9 mosaicism case described in this report, the main disease features displayed by the child were dysmorphic craniofacial

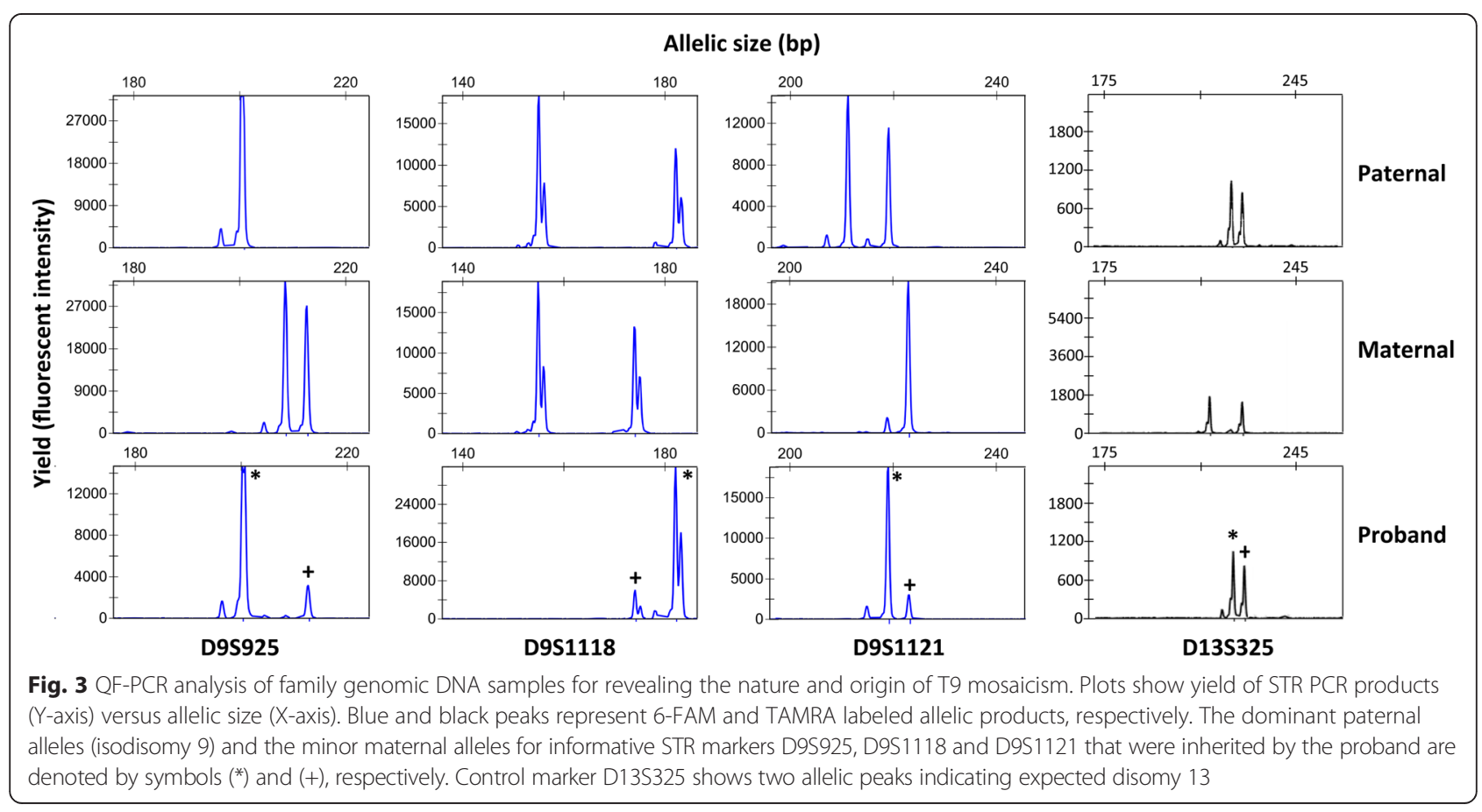


features, developmental delay, congenital cerebral dysplasia and congenital heart disease. However, in contrast to other children with a similar low level of T9 mosaicism [11], growth retardation and developmental delay was particularly severe in this 22 month old girl and constant daily care was needed.

Intriguingly, from all the children examined with T9 mosaicism to date, there appears to be no clear correlation of the level of mosaicism with the severity of the disease phenotype [11]. One plausible explanation to reconcile this lack of phenotypic/genotypic correlation is that the level of T9 mosaicism has only generally been assessed in peripheral blood lymphocytes and skin. It is possible that during pre- and post-natal development, the proportion of T9 cells in the various tissues has altered significantly due to differences in the intrinsic growth and apoptotic rates of the normal and trisomic cells. In addition, even within one tissue or organ, regional differences in T9 mosaicism may have also arisen. Three case reports provide evidence to support this notion. In the first case [12], prenatal diagnosis of amniocytes detected $100 \%$ T9 whereas postnatal analysis of kidney, skin, ovary and lung tissue from the deceased child who died at age 6 weeks revealed T9 mosaicism levels of $25 \%, 15.4 \%, 3.7 \%$ and $0 \%$, respectively. In two other cases that analysed fetuses that died in utero, there were also discrepancies noted between the level of T9 mosaicism in different tissues $[13,14]$. Similarly, in the case presented here, we observed $30 \%$ mosaicism from placental tissue in the prenatal period by NIPT, but only $17 \%$ and $19 \%$ respectively, in blood and buccal cells by interphase FISH.

Extensive data collected from pre-implantation genetic diagnosis cycles [15] demonstrates that chromosomal trisomy arising in the somatic tissue of an individual has an early developmental origin. Several mechanisms have been proposed for the formation of mosaicism including either a gametal meiotic I/meiotic II chromosome nondisjunction error and a subsequent trisomy rescue by anaphase lag in the dividing pre-implantation embryo or mitotic non-disjunction in one of the early cell divisions of the cleavage stage embryo [16]. Through these mechanisms, the formation of trisomy mosaicism in association with uniparental disomy (UPD) is also another possible outcome [17]. Mosaic embryos can continue to develop throughout the pre- and post implantation period leading to the formation of diploid-aneuploid mosaic fetuses. In this case study, Chr9 STR analyses of the family pedigree revealed that the proband had inherited two identical copies of paternal Chr9 (isodisomy 9) and one copy of maternal Chr9 with comparative paternal:maternal Chr9 ratios of approximately 10:1. On this basis, we conclude that the origin of the T9 mosaicism in the child was most likely due to a paternal meiotic II non-disjunction error of $\mathrm{Chr} 9$ followed by a post-zygotic
T9 rescue event of the maternally inherited Chr9 in the initial cleavage divisions of the developing embryo. We speculate that these two independent Chr9 events in the pre-implantation period ultimately lead to the development of T9 mosaicism in the child, comprising paternal isodisomy 9 in the majority of somatic cells and T9 in the remaining somatic cells.

From the literature, there have several prenatal reports $[18,19]$ where the fetus has been identified with T9 mosaicism in association with maternal UPD 9. Based on records of all documented UPD 9 cases (http://upd-tl.com/ upd.html), there have been 10 births with maternal UPD 9 and one birth with paternal UPD 9. Clinical examination of those individuals with maternal UPD 9, which is associated with maternally imprinted genes, revealed no significant disease phenotypes [20]. However, when the maternal UPD9 was also associated with T9, the patients displayed typical symptoms characteristic of trisomy 9 mosaicism syndrome. The one case of paternal UPD 9 was also found in the context of trisomy 9 mosaicism with the child exhibiting typical clinical symptoms of trisomy 9 mosaicism syndrome [21]. Thus the child reported in this study represents the second individual identified with paternal UPD 9, which we identified as isodisomy 9. Based on the very severe developmental delay exhibited by this child, in addition to the possibility of variable levels of T9 in other tissues, we raise the possibility that recessive genes associated with growth and development on Chr9 such as DMRT1, NTRK2 and NTRKR2 [22] may have also contributed to the overall clinical disease phenotype of this child.

Over many years traditional prenatal diagnosis by karyotyping and FISH analysis of cultured amniocytes, in combination with ultrasound, has been relatively successful in identifying fetuses with T9 mosaicism [13, 23-25]. However, in clinical practice, decisions regarding termination of pregnancy are extremely difficult because several follow up studies have shown that the level of trisomy detected prenatally is often much lower in post-natal tissue $[12,26]$. In addition, it is almost impossible to determine the clinical significance of low-level trisomy in cultured amniocytes since it could represent either true fetal mosaicism or pseudomosaicism as a result of culture artifacts [27]. The case reported here was additionally confounding because the low-level T9 originally detected at 15 weeks gestation by NIPT was not identified by traditional confirmation by amniocentesis, cell culture and karyotyping even at 34 weeks gestation. This suggests that in this case, culturing of amniocytes may have caused preferential growth of normal cells over abnormal cells, masking the low level of T9 mosaicism. This case therefore highlights the need for direct analysis of the biopsied fetal cells without culture to increase detection sensitivity when lowlevel T9 mosaicism is suspected [28]. 


\section{Conclusions}

In summary, with increasing numbers of pregnant women undertaking NIPT as the primary test method for common fetal aneuploidies, this study shows the feasibility of also detecting a rare chromosomal disorder such as T9 mosaicism syndrome. Apart from T9 mosaicism, T8 mosaicism can also develop to term and cause severe disease phenotypes [29]. While reporting detection of these two rare chromosomal disorders diseases would extend the clinical utility of NIPT beyond common autosomal trisomies and sex aneuploidies, on a cautionary note, this may in turn increase the false positive rates since T8 and T9 are often associated with confined placental mosaicism [30]. Nevertheless NIPT, in conjunction with confirmatory invasive testing and ultrasound scanning, may ultimately prove a more robust approach to identify fetuses with these rare chromosome disease syndromes.

\section{Materials and methods}

\section{Noninvasive prenatal testing}

Clinical NIPT was performed according to previously described methods [5]. In brief, plasma DNA libraries were generated and subjected to massively parallel sequencing on the HiSeq2000 platform (Illumina, US) to generate $\sim 8$ million (M) 36 bp sequencing reads per sample. Using the Burrows Wheeler algorithm [31], 5 M reads were uniquely and perfectly aligned to the unmasked hg19 reference genome. After normalization and comparison to reference data, z-scores were assigned for each chromosome, with a normal range of $-3<\mathrm{z}<3$ for autosomal disomy.

\section{Cytogenetic studies}

Metaphase chromosomes were prepared from cultured PHA-stimulated peripheral blood cells. Karyotypes were determined from $\mathrm{G}$ banded metaphase chromosome analysis of 100 cells at a resolution of 320 bands. Interface FISH was performed on peripheral blood and buccal cells using 9p SpectrumGreen (locus 305 J7-T7) and 9q SpectrumOrange (locus D9S325) probes (TelVysion, Abbott Molecular, US) according to the recommended protocol. At least 100 cells were examined with $9 p$ and 9q probes to determine levels of Chr9 mosaicism.

\section{Quantitative fluorescent PCR analysis}

STR markers D9S157, D9S164, D9S288, D9S925, D9S1118, D9S1121, D9S1677, D9S1874 and D9S2157 with high heterozygosity indices $(>0.7)$ were selected for Chr9 analysis, with markers D21S1270 and DS13S325 serving as disomy controls. Forward primers for each STR marker were labeled with either 6-carboxy fluorescein (6FAM) or carboxy tetramethylrhodamine (TAMRA). Singleplex QF-PCR reactions (30 amplification cycles) were performed using $50 \mathrm{ng}$ of genomic DNA (Kapa Taq HotStart PCR kit, Kapa Biosystems) according to the manufacturer's instructions. Labeled PCR products were analyzed on a 3130 Genetic Analyzer (Applied Biosystems, US) and allelic peaks plotted as relative fluorescence intensity (Y-axis) versus size in nucleotides ( $\mathrm{X}$-axis).

\section{Consent}

Written informed consent was obtained from the parents of the child for the publication of this report and any accompanying images.

\section{Abbreviations}

NIPT: Noninvasive prenatal testing; QF-PCR: Quantitative fluorescent polymerase chain reaction; STR: Short tandem repeat; T: Trisomy; UPD: Uniparental disomy.

\section{Competing interests}

The authors declare that they have no competing interests.

\section{Authors' contributions}

$J M$, contributed to the cytogenetic studies, manuscript preparation and supervision of the study. DSC, contributed to manuscript preparation and technical review. JZ and LS, performed and interpreted the NIPT and QF-PCR experiments. HY, contributed to the cytogenetic studies and participated in patient review and management. HP, participated in patient review and critically reviewed the manuscript. All authors read and approved the final manuscript.

\section{Acknowledgments}

We thank the family for their participation in this research study, which aimed to understand the genetic basis of the severe developmental delay exhibited by their 22 month old daughter.

\section{Author details}

'Department of Obstetrics, Peking University First Hospital, No.1 Xianmen Street, Xicheng District, Beijing 100034, China. ${ }^{2}$ Berry Genomics, Building 9, No 6 Court Jingshun East Road, Chaoyang District, Beijing 100015, China.

Received: 24 March 2015 Accepted: 25 April 2015

Published online: 26 June 2015

\section{References}

1. Benn $\mathrm{P}, \mathrm{Cuckle} \mathrm{H}$, Pergament E. Non-invasive prenatal testing for aneuploidy: current status and future prospects. Ultrasound Obstet Gynecol. 2013;42:15-33.

2. Song Y, Liu C, Qi H, Zhang Y, Bian X, Liu J. Noninvasive prenatal testing of fetal aneuploidies by massively parallel sequencing in a prospective Chinese population. Prenat Diagn. 2013;33:700-6.

3. Bianchi DW, Parker RL, Wentworth J, Madankumar R, Saffer C, Das AF, et al. DNA sequencing versus standard prenatal aneuploidy screening. N Engl J Med. 2014;370:799-808.

4. American College of Obstetricians and Gynecologists Committee on Genetics. Committee opinion No. 545: noninvasive prenatal testing for fetal aneuploidy. Obstet Gynecol. 2012;120:4-1532.

5. Liang D, Lv W, Wang H, Xu L, Liu J, Li H, et al. Non-invasive prenatal testing of fetal whole chromosome aneuploidy by massively parallel sequencing. Prenat Diagn. 2013;33:409-15.

6. Song $Y$, Huang S, Zhou X, Jiang $Y$, Qi Q, Bian X, et al. Non-invasive prenatal testing for fetal aneuploidies in the first trimester of pregnancy. Ultrasound Obstet Gynecol. 2015;45:55-60.

7. ISCN 2013. An International System for Human Cytogenetic Nomenclature (2013). Editors: L.g. Shaffer, J McGowen, M. Schmid. Publisher: Karger, Basel, Switzerland.

8. Feingold M, Atkins L. A case of trisomy 9. J Med Genet. 1973;10:184-7.

9. Haslam RH, Broske SP, Moore CM, Thomas GH, Neill CA. Trisomy 9 mosaicism with multiple congenital anomalies. J Med Genet. 1973;10:180-4.

10. Arnold GL, Kirby RS, Stern TP, Sawyer JR. Trisomy 9: review and report of two new cases. Am J Med Genet. 1995;56:252-7. 
11. Bruns D. Presenting physical characteristics, medical conditions, and developmental status of long-term survivors with trisomy 9 mosaicism. Am J Med Genet A. 2011;155A:1033-9.

12. Sherer DM, Wang $\mathrm{N}$, Thompson HO, Peterson JC, Miller ME, Metlay LA, et al. An infant with trisomy 9 mosaicism presenting as a complete trisomy 9 by amniocentesis. Prenat Diagn. 1992;12:31-7.

13. Pfeiffer RA, Ulmer R, Kniewald A, Wagner-Thiessen E. Prenatal diagnosis of trisomy 9 mosaicism possibly limited to fetal membranes. Prenat Diagn. 1984:4:387-9.

14. Smoleniec JS, Davies T, Lunt P, Berry PJ, James D. Complex mosaicism associated with trisomy 9. Prenat Diagn. 1993;13:211-3.

15. Harper JC, Wilton L, Traeger-Synodinos J, Goossens V, Moutou C, SenGupta SB, et al. The ESHRE PGD consortium: 10 years of data collection. Hum Reprod Update. 2012;18:234-47.

16. Taylor TH, Gitlin SA, Patrick JL, Crain JL, Wilson JM, Griffin DK. The origin, mechanisms, incidence and clinical consequences of chromosomal mosaicism in humans. Hum Reprod Update. 2014:20:571-81.

17. Eggermann T, Soellner L, Buiting K, Kotzot D. Mosaicism and uniparental disomy in prenatal diagnosis. Trens Mol Med. 2015;21:77-87.

18. Wilkinson TA, James RS, Crolla JA, Cockwell AE, Campbell PL, Temple IK. A case of maternal uniparental disomy of chromosome 9 in association with confined placental mosaicism for trisomy 9. Prenat Diagn. 1996;16:371-4.

19. Slater HR, Ralph A, Daniel A, Worthington S, Roberts C. A case of maternal uniparental disomy of chromosome 9 diagnosed prenatally and the related problem of residual trisomy. Prenat Diagn. 2000;20:930-2.

20. Morison IM, Reeve AE. A catalogue of imprinted genes and parent-of-origin effects in humans and animals. Hum Mol Genet. 1998;7:1599-609.

21. Van der Hagen CB, Eiklid K, Orstavik KH, Braaten O, Prescott T, Kroken M, et al. Prenatally diagnosed trisomy 9 mosaicism and paternal uniparental disomy 9 in the child. Europ J Hum Genet. 2003;11(Suppl):409.

22. Cooper GM, Coe BP, Girirajan S, Rosenfeld JA, Vu TH, Baker C et al. A copy number variation morbidity map of developmental delay. Nat Genet. 2011:43:838-46.

23. Zadeh TM, Peters J, Sandlin C. Prenatal diagnosis of mosaic trisomy 9. Prenat Diagn. 1987;7:67-70

24. Schwartz S, Ashai S, Meijboom EJ, Schwartz MF, Sun CC, Cohen MM Prenatal detection of trisomy 9 mosaicism. Prenat Diagn. 1989;9:549-54.

25. Merino A, De Perdigo A, Nombalais F, Yvinec M, Le Roux MG, Bellec V. Prenatal diagnosis of trisomy 9 mosaicism: two new cases. Prenat Diagn. 1993;13:1001-7.

26. Kosaki R, Hanai S, Kakishima H, Okada MA, Hayashi S, Ito $Y$, et al. Discrepancies in cytogenetic results between amniocytes and postnatally obtained blood: trisomy 9 mosaicism. Congenit Anom (Kyoto). 2006;46:115-7.

27. Daniel A, Wu Z, Darmanian A, Malafiej P, Tembe V, Peters G, et al. Issues arising from the prenatal diagnosis of some rare trisomy mosaics-the importance of cryptic fetal mosaicism. Prenat Diagn. 2004;24:524-36.

28. Pham J, Shaw C, Pursley A, Hixson P, Sampath S, Roney E, et al. Somatic mosaicism detected by exon-targeted, high-resolution aCGH in 10,362 consecutive cases. Eur J Hum Genet. 2014;22:969-78.

29. Berry AC, Mutton DE, Lewis DG. Mosaicism and the trisomy 8 syndrome. Clin Genet. 1978;14:105-14

30. Wolstenholme J. Confined placental mosaicism for trisomies 2, 3, 7, 8, 9, 16, and 22: their incidence, likely origins, and mechanisms for cell lineage compartmentalization. Prenat Diagn. 1996:16:511-24.

31. Li H, Durbin R. Fast and accurate short read alignment with burrows- wheeler transform. Bioinformatics. 2009:25:1754-60.

\section{Submit your next manuscript to BioMed Central and take full advantage of:}

- Convenient online submission

- Thorough peer review

- No space constraints or color figure charges

- Immediate publication on acceptance

- Inclusion in PubMed, CAS, Scopus and Google Scholar

- Research which is freely available for redistribution

Submit your manuscript at www.biomedcentral.com/submit 